\section{(D) Check for updates}

Cite this: Analyst, 2020, 145, 3757

\title{
Correction: Prospective on using fibre mid- infrared supercontinuum laser sources for in vivo spectral discrimination of disease
}

\author{
Angela B. Seddon, ${ }^{\star a}$ Bruce Napier, ${ }^{\mathrm{b}}$ Ian Lindsay,,$^{\mathrm{c}}$ Samir Lamrini, ${ }^{\mathrm{d}}$ \\ Peter M. Moselund, ${ }^{e}$ Nicholas Stone, ${ }^{f}$ Ole Bang ${ }^{g}$ and Mark Farries ${ }^{h}$
}

DOI: 10.1039/c9an90110h

rsc.li/analyst

Correction for 'Prospective on using fibre mid-infrared supercontinuum laser sources for in vivo spectral discrimination of disease' by Angela B. Seddon et al., Analyst, 2018, 143, 5874-5887.

The authors regret that the figure caption for Fig. 8 in the original article is incorrect. The attribution of the synchrotron data in Fig. 8 to the performance parameters of beamline B22 of the Diamond Synchrotron Light Source was incorrect. The true source of the data shown is a calculation based on previously published models for synchrotron spectral radiance. The data therefore have no connection to Diamond other than insofar as these data are representative of the performance of a generic synchrotron. No data obtained from Diamond, publications by Diamond users or material published online by Diamond were used to produce Fig. 8 in the paper. Nor were the data calculated to be specifically representative of Diamond B22 performance.

The correct version of the caption for Fig. 8 is as shown below.

Fig. 8 - Comparison of power spectral density (PSD) available in a diffraction-limited beam for various MIR sources. Compared are: blackbody thermal emitter at $1500 \mathrm{~K}$ e.g. common GloBar® sources; synchrotron bending magnet output, calculated for $0.5 \mathrm{~A}$ beam current, ${ }^{36}$ captured within a diffraction-limited solid angle; cartoon of typical quantum cascade laser array output (representative of data from ref. 35). Note that QCLs at wavelengths as low as $3 \mu \mathrm{m}$ are now available); cartoon approximating the PSD of the most powerful wideband SIF MIR-SC to date $;^{31}$ commercial fibre MWIR SC system ${ }^{34}$ and recently reported tapered chalcogenide photonic crystal fibre SC. ${ }^{32}$ Also shown are the non-diffraction-limited PSD for a $1500 \mathrm{~K}$ blackbody emitter imaged using a range of commercial reflective microscope objectives with magnifications of $15 \times, 25 \times$ and $74 \times$ (figure redrawn and amended from ref. 33).

Consequently, sections of the text in the manuscript should be adjusted according to this change, and these are detailed below.

The paragraph on page 5880 beginning "Taking the SIF (step-index-fibre) MIR-SC..." should be correctly given as "Taking the SIF (step-index-fibre) MIR-SC with best broadband power spectral density (PSD) from 2.2-12 $\mu \mathrm{m}$ now achieved ${ }^{31}$ (see Table 1), Fig. 8 (compiled in MINERVA) compares existing MIR sources ${ }^{33}$ emitting into a diffraction-limited beam. It can be seen from Fig. 8 that commercial fibre MWIR-SC covering $\sim 3-5 \mu \mathrm{m}$ (ref. 34 ) are as spectrally bright as the longer wavelength quantum cascade lasers. ${ }^{35}$ Also compared are the calculated PSD of wideband blackbody thermal emitters (e.g. GloBar®) and representative synchrotron (SR) MIR output calculated for a $0.5 \mathrm{~A}$ beam current. ${ }^{36}$ Fig. 8 demonstrates that, when collecting radiation at the diffraction limit, fibre MIR-SC lasers have now been realised that yield several times the PSD of synchrotron-generated MIR beams over the broad ranges of spectroscopic significance represented here. It should be noted that typical synchrotron radiation extends far beyond the MWIR molecular fingerprint region of interest here. Fig. 8 also illustrates that while not having a PSD as

\footnotetext{
${ }^{a}$ Mid-Infrared Photonics Group, George Green Institute for Electromagnetics' Research, Faculty of Engineering, University of Nottingham, NG7 2RD, UK.

E-mail: angela.seddon@nottingham.ac.uk

${ }^{b}$ Vivid Components Ltd, German Office, Dr-Rörig-Damm 22, 33102 Paderborn, Germany

${ }^{c}$ Gooch \& Housego, UK

${ }^{d}$ LISA laser products OHG, Fuhrberg \& Teichmann, Albert Einstein Straße, 1-9 37191 Katlenburg-Lindau, Germany

${ }^{e}$ NKT Photonics A/S, Blokken 84, DK-3460 Birkerød, Denmark

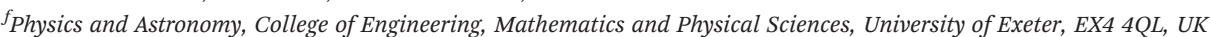

${ }^{g}$ Fibre Sensors and Supercontinuum Group, DTU Fotonik, Technical University of Denmark, DK-2800 Kongens Lyngby, Denmark

${ }^{h}$ Gooch \& Housego (Torquay), Broomhill Way, Torquay, TQ2 7QL, UK

$\dagger$ Now at: H. H. Wills Physics Laboratory, Tyndall Ave., University of Bristol, BS8 1TL, UK.
} 
high as an array of MIR-QCLs, MIR-SC sources cover several times the spectral range with a single device. Fig. 8, also shows the total PSD from a blackbody emitter imaged through typical reflective MIR microscope objectives of magnification: $15 \times, 25 \times$ and $74 \times$. This optical configuration does not yield a diffraction-limited output, but emphasises that the clear advantage of high-radiance sources like a supercontinuum is maximised for point-scanned or stand-off spectral imaging."

As a result of this change to both the caption of Fig. 8 and the text, the authors would like to replace ref. 36 with the reference listed below as ref. 1 .

The Royal Society of Chemistry apologises for these errors and any consequent inconvenience to authors and readers.

\section{References}

1 P. Dumas and L. Miller, Biological and Biomedical Applications of Synchrotron Infrared Microspectroscopy, J. Biol. Phys., 2003, 29(2-3), 201-218. 